IASSNS-HEP-95/12

McGill/95-10

hep-th/9503055

published in Phys. Rev. Lett. 74 (1995) 4767

February 1995

\title{
String Theory, Misaligned Supersymmetry, and the Supertrace Constraints
}

\author{
Keith R. Dienes ${ }^{1}\left[\right.$, Moshe Moshe ${ }^{2}$ t, and Robert C. Myers 3 尚 \\ ${ }^{1}$ School of Natural Sciences, Institute for Advanced Study \\ Olden Lane, Princeton, NJ 08540 USA \\ ${ }^{2}$ Department of Physics, Technion - Israel Inst. of Technology \\ Haifa 32000, Israel \\ ${ }^{3}$ Department of Physics, McGill University \\ 3600 University St., Montréal, Québec H3A-2T8 Canada
}

\begin{abstract}
We demonstrate that string consistency in four spacetime dimensions leads to a spectrum of string states which satisfies the supertrace constraints Str $\mathbf{1}=0$ and $\operatorname{Str} M^{2} \propto \Lambda$ at tree level, where $\Lambda$ is the one-loop string cosmological constant. This result holds for a large class of string theories, including critical heterotic strings. For strings lacking spacetime supersymmetry, these supertrace constraints will be satisfied as a consequence of a hidden "misaligned supersymmetry" in the string spectrum. These results thus severely constrain the possible supersymmetry-breaking scenarios in string theory, and suggest a new intrinsically stringy mechanism whereby such supertrace constraints may be satisfied without phenomenologically unacceptable consequences.
\end{abstract}

\footnotetext{
*E-mail address: dienes@sns.ias.edu.

${ }^{\dagger}$ E-mail address: phr74mm@vmsa.technion.ac.il

$\ddagger$ E-mail address: rcm@hep.physics.mcgill.ca
} 
In quantum field theories with broken supersymmetry, the divergence properties of amplitudes are governed by the values of various supertraces calculated over the particles in the resulting spectrum. For example, in four-dimensional spacetime, $\operatorname{Str} M^{4}$ controls the logarithmic divergences in the vacuum energy density, while $\operatorname{Str} M^{2}$ and Str $M^{0} \equiv$ Str 1 control the quadratic and quartic divergences respectively. If the supersymmetry (SUSY) is unbroken, each of these supertraces of course vanishes as a consequence of strict level-by-level degeneracies between bosonic and fermionic degrees of freedom. It is phenomenologically important, however, to construct nonSUSY field theories which retain the soft divergence behavior of their SUSY counterparts, hopefully cancelling the quartic and quadratic divergences which might appear. As is well-known, this can be achieved at tree level by breaking the SUSY either spontaneously, or through the addition of certain "soft" breaking terms; indeed, in many cases the vanishing of Str 1 and Str $M^{2}$ is preserved. The problem with these scenarios, however, is that they satisfy these two constraints in a multiplet-by-multiplet fashion, so that the mass of each state in the broken theory is constrained to be relatively close to that of its former superpartner. Since this is unacceptable from a phenomenological standpoint, one must therefore rely on further quantum effects in order to lift these constraints. One then finds that although Str 1 continues to vanish, Str $M^{2}$ takes a non-zero, model-dependent value.

In this paper we consider the corresponding situation in string theory, and find that at tree level, the general requirements of string consistency lead to similar supertrace constraints. Specifically, defining our string-theoretic supertraces as

$$
\operatorname{Str} M^{2 \beta} \equiv \lim _{\gamma \rightarrow 0}\left\{\sum_{\text {states }}(-1)^{F}\left(M_{i}\right)^{2 \beta} e^{-\gamma M_{i}^{2}}\right\},
$$

we find that for a large class of tachyon-free string theories in four dimensions,

$$
\text { Str } \mathbf{1}=0 \quad \text { and } \quad \operatorname{Str} M^{2}=-\frac{3}{4 \pi^{2}} \Lambda_{\text {string }}
$$

where $\Lambda_{\text {string }}$ is the corresponding (finite) one-loop string-theoretic cosmological constant. Thus, the spacetime bosons and fermions at all string mass levels must always arrange themselves at tree level so that these two supertrace constraints are satisfied. Unlike the case in field theory, however, we will find that these results rely on only the general properties of string consistency (in particular, the presence of modular invariance and the absence of physical tachyons). These results are therefore independent of the particular string model in question, and consequently have broader applicability than in field theory.

A second and perhaps more important difference concerns the manner in which these constraints are satisfied. In string theories with spacetime SUSY, $\Lambda_{\text {string }}=0$ and these constraints are trivially satisfied through an exact boson/fermion degeneracy. This is just as in the field theory case. However, for string theories without spacetime SUSY, these constraints need not be satisfied multiplet-by-multiplet. Rather, as we 
will discuss, these constraints are generally satisfied in a different manner, through a so-called "misaligned SUSY". Misaligned SUSY therefore represents an entirely new stringy scenario whereby constraints such as those in Eq. (2) may be satisfied without phenomenologically unacceptable consequences. As we shall see, this alternative scenario is possible in string theory because of the existence of an infinite tower of string states, thereby permitting the freedom to satisfy the supertrace constraints across the entire string spectrum, rather than multiplet-by-multiplet. Indeed, in nonSUSY string models it is not necessary (or often even possible) to make reference to a (broken) multiplet structure in the spectrum. The bosonic and fermionic states which appear will nevertheless conspire to exhibit a "misaligned SUSY" and satisfy Eq. (2) in a highly non-trivial manner.

Let us begin by first reviewing the appearance of the supertrace constraints in field theory. Perhaps the simplest manner in which they arise is through the calculation of the field-theoretic vacuum energy density (cosmological constant), given to lowest order as

$$
\begin{aligned}
\Lambda_{\text {field }} & =\frac{1}{2} \sum_{i}(-1)^{F} \int \frac{d^{D} p}{(2 \pi)^{D}} \log \left(p^{2}+M_{i}^{2}\right) \\
& =-\frac{1}{2} \sum_{i}(-1)^{F} \int \frac{d^{D} p}{(2 \pi)^{D}} \int_{0}^{\infty} \frac{d t}{t} e^{-\left(p^{2}+M_{i}^{2}\right) t} \\
& =-\frac{1}{2} \frac{1}{(4 \pi)^{D / 2}} \sum_{i}(-1)^{F} \int_{0}^{\infty} \frac{d t}{t^{1+D / 2}} e^{-M_{i}^{2} t} .
\end{aligned}
$$

Here the summations are over all states in the theory (with corresponding masses $M_{i}$ ), and we have kept the spacetime dimension $D$ arbitrary. In the second line we have passed to a Schwinger proper-time representation wherein any ultraviolet divergences from $p^{\mu} \rightarrow \infty$ appear as a divergence as $t \rightarrow 0$, while infrared divergences appear as $t \rightarrow \infty$.

We are concerned with the divergence properties of $\Lambda_{\text {field }}$, and from Eq. (3) these can now easily be determined. The absence of any infrared divergence from the $t \rightarrow \infty$ region is guaranteed if there are no tachyonic states with $M_{i}^{2}<0$. Ultraviolet divergences, on the other hand, would appear as $t \rightarrow 0$. These will therefore be absent if

$$
\sum_{i}(-1)^{F} e^{-M_{i}^{2} t} \sim t^{\alpha} \text { with } \alpha>D / 2
$$

as $t \rightarrow 0$. Since we are in the $t \rightarrow 0$ limit, we can expand the exponential, $e^{-M_{i}^{2} t}=1-$ $M_{i}^{2} t+M_{i}^{4} t^{2} / 2+\ldots$, and thereby obtain the separate supertrace conditions $\operatorname{Str} M^{2 \beta} \equiv$ $\sum_{i}(-1)^{F}\left(M_{i}\right)^{2 \beta}=0$, valid for $\beta=0,1, \ldots,[D / 2]$ where $[x]$ denotes the greatest integer less than or equal to $x$. In particular, for $D=4$, this yields the three separate supertrace constraints $\operatorname{Str} \mathbf{1}=\operatorname{Str} M^{2}=\operatorname{Str} M^{4}=0$, with logarithmic, quadratic, and quartic divergences respectively if the $\operatorname{Str} M^{4}, \operatorname{Str} M^{2}$, and $\operatorname{Str} 1$ conditions are not satisfied. While all three of these supertrace conditions are satisfied in SUSY theories, 
spontaneous or soft SUSY-breaking preserves only the Str 1 (and occasionally the Str $M^{2}$ ) condition at tree level [1].

Let us now consider the corresponding situation in string theory. A priori, there are three fundamental differences. The first is that in string theory, there are an infinite number of states; these generally appear in towers whose levels are integerspaced (in Planck-scale units), and whose state degeneracies grow exponentially with mass. This is why a regulator such as that in Eq. (11) must be chosen. The second difference is that whereas field-theoretic states are characterized by a single mass $M_{i}$, in string theory the energy of each state is described through two such quantities, the separate left- and right-moving mass contributions $M_{i}^{(L)}$ and $M_{i}^{(R)}$ whose squares always differ by integers. A state is deemed "physical" if $M_{i}^{(L)}=M_{i}^{(R)}$, and "unphysical" otherwise; note that only the physical string states correspond to actual particles in spacetime. Nevertheless, both types of states contribute to the stringtheoretic one-loop cosmological constant $\Lambda_{\text {string. }}$. Indeed, in string theory, $\Lambda_{\text {string }}$ is given by

$$
\Lambda_{\text {string }} \equiv \int_{\mathcal{F}} \frac{d^{2} \tau}{(\operatorname{Im} \tau)^{2}} Z(\tau)
$$

where the integration variable $\tau$ is the torus complex modular parameter, and where the string partition function $Z(\tau)$ is a trace over the Fock space of physical and unphysical string states,

$$
Z(\tau)=(\operatorname{Im} \tau)^{1-D / 2} \sum_{\text {states }}(-1)^{F} q^{\left[M_{i}^{(L)}\right]^{2}} \bar{q}^{\left[M_{i}^{(R)}\right]^{2}}
$$

with $q \equiv e^{2 \pi i \tau}$ and with all masses in units of the Planck mass. In the usual string formulation, the modular invariance of $Z(\tau)$ allows one to truncate the region of $\tau$ integration, as in Eq. (5), to the fundamental domain of the modular group, $\mathcal{F} \equiv$ $\left\{\tau:|\tau|^{2} \geq 1, \operatorname{Im} \tau>0,|\operatorname{Re} \tau| \leq 1 / 2\right\}$.

Since $\tau_{2} \equiv \operatorname{Im} \tau$ in string theory plays the role of the Schwinger proper time $t$ in field theory, we see that the region $\tau_{2} \rightarrow \infty$ corresponds to the infrared, and $\tau_{2} \rightarrow 0$ to the ultraviolet. Infrared divergences will thus be absent, as in field theory, if there are no physical tachyonic states with $\left[M_{i}^{(L)}\right]^{2}=\left[M_{i}^{(R)}\right]^{2}<0$. Indeed, this is part of what defines a physically consistent string theory. Turning to the ultraviolet, however, we see that the truncation of the region of $\tau$-integration to the fundamental domain $\mathcal{F}$ has already excluded the region near $\tau_{2} \rightarrow 0$. This is the root of the wellknown remarkable ultraviolet finiteness properties of string theory, and the symmetry by which this occurs (namely, modular invariance) is also part of what defines a consistent string theory.

However, it is this feature which represents the third fundamental difference between string theory and field theory, for we see that the ultraviolet finiteness of $\Lambda_{\text {string }}$ has automatically arisen through a truncation in the range of integration. What we require, however, is an alternative expression for $\Lambda_{\text {string }}$ whose finiteness explicitly rests on the behavior of the string spectrum. Indeed, it is only in this way that we 
can exploit the finiteness of $\Lambda_{\text {string }}$ to derive a series of supertrace mass formulas for string theory just as exist in field theory. Fortunately, for a large class of tachyon-free string theories, such an alternative expression exists [2]:

$$
\Lambda_{\text {string }}=\frac{\pi}{3} \lim _{\tau_{2} \rightarrow 0} \int_{-1 / 2}^{1 / 2} d \tau_{1} Z(\tau)
$$

where $\tau_{1} \equiv \operatorname{Re} \tau$. This class includes all unitary non-critical strings, critical Type-II strings, as well as the phenomenologically interesting case of $D>2$ critical heterotic strings. . Substituting the form of the string partition function $Z(\tau)$ in Eq. (6) and explicitly performing the $\tau_{1}$ integral, we then obtain

$$
\Lambda_{\text {string }}=\frac{\pi}{3} \lim _{\tau_{2} \rightarrow 0}\left(\tau_{2}\right)^{1-D / 2} \sum_{\text {states }}(-1)^{F} e^{-4 \pi \tau_{2}\left[M_{i}^{(L)}\right]^{2}} \delta_{M_{i}^{(L)}, M_{i}^{(R)}} .
$$

We thus see that in this formulation, only the masses of the physical string states are relevant. Defining $M_{i} \equiv M_{i}^{(L)}=M_{i}^{(R)}$, we therefore have

$$
\Lambda_{\text {string }}=\frac{\pi}{3} \lim _{\tau_{2} \rightarrow 0}\left(\tau_{2}\right)^{1-D / 2} \sum_{\substack{\text { phys. } \\ \text { states }}}(-1)^{F} e^{-4 \pi \tau_{2} M_{i}^{2}},
$$

so that $\Lambda_{\text {string }}$ is indeed free of ultraviolet divergences if and only if, as $\tau_{2} \rightarrow 0$,

$$
\sum_{\substack{\text { phys. } \\ \text { states }}}(-1)^{F} e^{-4 \pi \tau_{2} M_{i}^{2}} \sim\left(\tau_{2}\right)^{\alpha} \quad \text { with } \quad \alpha \geq D / 2-1 .
$$

At this point we have shown that the masses of physical states throughout the spectra of such consistent tachyon-free string theories must always arrange themselves so as to satisfy Eq. (10). This result, however, is completely analogous to the corresponding field-theoretic result in Eq. (14), and the different powers of $t$ or $\tau_{2}$ which appear on the right sides of these equations reflect the extra finiteness properties of string theory relative to field theory (with the quartic and quadratic divergences in field theory corresponding respectively to logarithmic divergences and constant terms in string theory). It is therefore tempting to proceed as for the field-theoretic case, and expand the exponential to obtain the corresponding supertraces. However, in the string case it is not technically proper to expand the exponential before taking the limit, since our Fock space of string states is infinite-dimensional. Rather, rigorously defining our string supertraces as in Eq. (1) and identifying $\gamma=4 \pi \tau_{2}$, we should properly evaluate these supertraces without expanding the exponentials, but rather

* Note that the critical heterotic case is special due to the appearance of unphysical tachyons with $\left[M^{(L)}\right]^{2}=-1,\left[M^{(R)}\right]^{2}=0$. However, for $D>2$, one can circumvent this difficulty by compactifying to a box of $(D-2)$-dimensional volume $V$, and taking $V \rightarrow \infty$ after the calculations are performed. See Ref. [2] for further details. We thank D. Kutasov for discussions on this point. 
by taking derivatives with respect to $\tau_{2}$ only after the summation is performed:

$$
\begin{aligned}
\operatorname{Str} M^{2 \beta} & =\lim _{\tau_{2} \rightarrow 0}\left\{\left(\frac{-1}{4 \pi} \frac{d}{d \tau_{2}}\right)^{\beta} \sum_{i}(-1)^{F} e^{-4 \pi \tau_{2} M_{i}^{2}}\right\} \\
& =\lim _{\tau_{2} \rightarrow 0}\left\{\left(\frac{-1}{4 \pi} \frac{d}{d \tau_{2}}\right)^{\beta}\left[\frac{3}{\pi} \Lambda_{\text {string }} \tau_{2}{ }^{D / 2-1}\right]\right\} .
\end{aligned}
$$

This yields, however, the same results as we would have obtained by expanding the exponentials. In particular, we find from Eq. (11) that for general $D$,

$$
\operatorname{Str} M^{2 \beta}=0 \text { for } \beta<D / 2-1, \beta \in \mathbb{Z},
$$

while for even $D$ we also have the result

$$
\operatorname{Str} M^{D-2}=\frac{3}{\pi} \frac{(D / 2-1) !}{(-4 \pi)^{D / 2-1}} \Lambda_{\text {string }} .
$$

Thus, for $D=4$, we find that the spectra of all consistent unitary non-critical strings and critical Type-II and heterotic strings must satisfy the supertrace constraints in Eq. (2).

We emphasize that our derivation has exploited only the fundamental characteristics of string consistency - namely, the absence of physical tachyons and the existence of modular invariance. We have not imposed the finiteness of $\Lambda_{\text {string }}$ as is done in field theory for $\Lambda_{\text {field }}$; rather, the finiteness of $\Lambda_{\text {string }}$ is a consequence of these more fundamental properties. Hence the string case differs quite markedly from the field-theoretic case. For example, while the vast majority of non-SUSY field theories do not obey any sort of supertrace conditions, we see that it is generally impossible to avoid these constraints in string theory. They are indeed generic properties of the moduli space of such tree-level non-SUSY string vacua.

Given these results, let us now discuss how string theory manages to evade the phenomenologically undesirable consequences which would arise in field theory. It is here that the existence of an infinite number of string states proves crucial. Indeed, it has recently been shown [3] that the spectrum of any consistent string theory which is modular invariant and free of physical tachyons will necessarily exhibit a so-called "misaligned SUSY". In the case of non-SUSY strings, this hidden symmetry takes the form of a subtle boson/fermion oscillation in which, for example, any surplus of bosons at any given string level necessarily implies a larger surplus of fermions at a higher level, which in turn implies an even larger boson surplus at an even higher level, and so forth throughout the infinite tower of states. Such behavior is sketched in Fig. 1 for a simple string theory containing two sectors, a bosonic sector with states at integer levels $M^{2}$ (in units of the Planck mass $M_{0}^{2}$ ), and a fermionic sector with states at levels $M^{2} \in \mathbb{Z}+1 / 2$. The numbers $g_{M}$ of bosonic minus fermionic states at each level $M$ are indicated by the solid dots. Although 


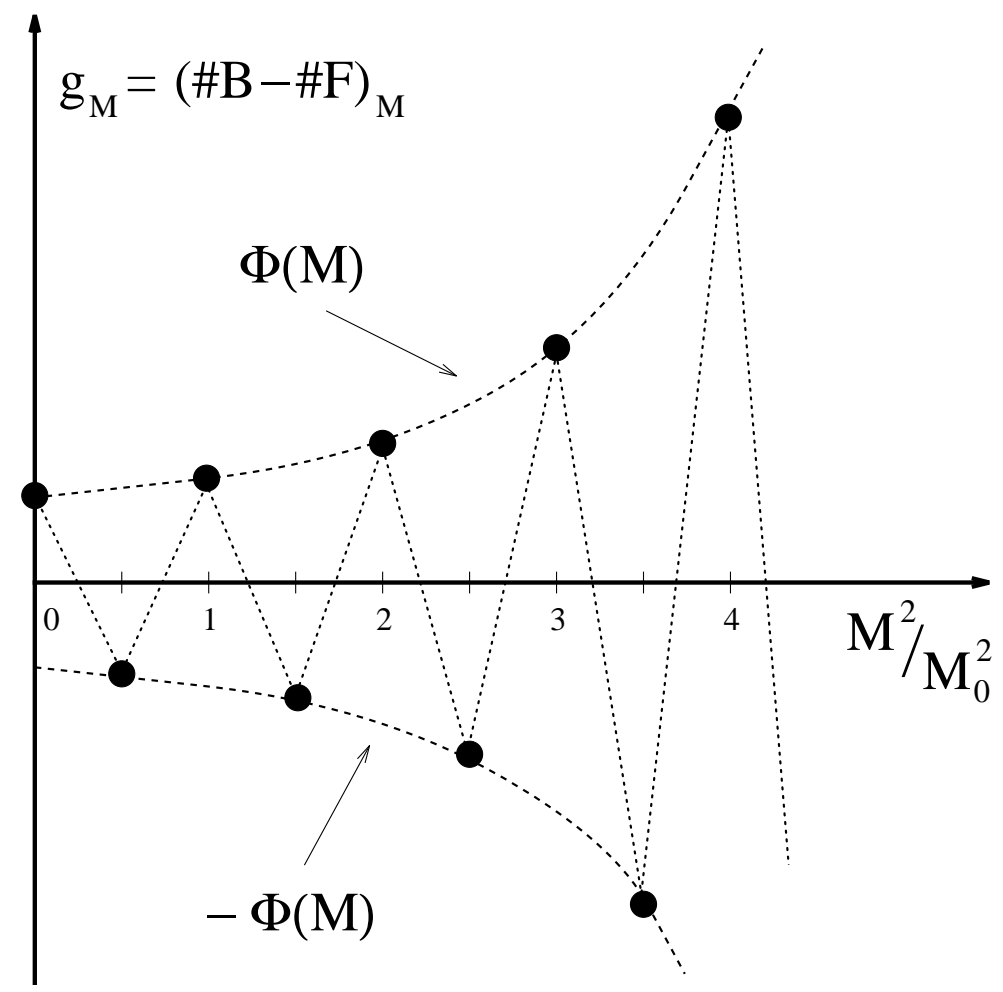

Figure 1: Misaligned SUSY and the resulting boson/fermion oscillations.

these two sectors are "misaligned" by a half-unit of energy, the number of such states at each level always grows exponentially according to complicated functions $\Phi(M)$ which are exactly equal and opposite for the two sectors. Even for string theories containing many sectors, the sum of the corresponding functional forms $\sum_{i} \Phi_{i}(M)$ over all string sectors must always cancel, and similar oscillations will appear. Further details behind this "misaligned SUSY" can be found in Ref. [3].

It is easy to check that degeneracies $g_{M}$ which behave in this oscillatory fashion will yield vanishing Str 1 when regulated as in Eq. (11). This type of stringy boson/fermion oscillation is therefore precisely what enables a string spectrum with exponentially growing numbers of string states to satisfy our supertrace constraints. Moreover, since such oscillations achieve cancellations between states at different energy levels across the infinite string spectrum, no strict multiplet-by-multiplet cancellations are necessary or even occur. Indeed, it is possible to construct consistent non-SUSY string models whose massless (observable) states are those of the Standard Model, but whose (broken) superpartners are either absent or at the Planck scale [6]. The spectra of such theories will nevertheless satisfy our supertrace constraints.

We conclude with some final comments. First, we observe that this "misaligned SUSY" mechanism can even be applied directly in field theory, since this stringinspired oscillation scenario does not depend on the particular scale $M_{0}^{2}$ of the level- 
spacing. Indeed, all that is required for the absence of such divergences is the cancellation of the degeneracy functions $\Phi(M)$. Thus, for example, it may be possible to exploit this mechanism to build an alternate non-supersymmetric solution to the gauge hierarchy problem.

Second, motivated by these supertrace results, we may ask whether there exist non-SUSY string theories which nevertheless have vanishing $\Lambda_{\text {string }}$. Indeed, such points in string moduli space would lead to string spectra satisfying both Str $1=\operatorname{Str} M^{2}=0$, thereby ensuring at most logarithmic divergences in any field theory containing the same numbers and energy distribution of bosonic and fermionic states. Furthermore, such points would have vanishing dilaton one-point functions, as required for vacuum stability at one loop and finite string amplitudes at higher loops. Unfortunately, despite various efforts [4], no non-SUSY models with vanishing $\Lambda_{\text {string }}$ have yet been constructed. There do exist, however, string-like partition functions $Z(\tau)$ which are non-vanishing (i.e., non-supersymmetric), but whose one-loop integrals $\Lambda$ vanish exactly [5]. Thus, there exist known non-supersymmetric distributions $\left\{g_{M}\right\}$ of bosonic and fermionic states which lead to vanishing $\Lambda_{\text {string }}$, and for which both Str 1 and Str $M^{2}$ cancel non-trivially. Moreover, we see from Eq. (12) that one can also obtain such $\left\{g_{M}\right\}$ with vanishing supertraces by considering nonSUSY strings in higher dimensions. For example, the degeneracies $\left\{g_{M}\right\}$ from the $D=10$ non-SUSY tachyon-free $S O(16) \otimes S O(16)$ string have $\operatorname{Str} \mathbf{1}, \operatorname{Str} M^{2}, \operatorname{Str} M^{4}$, and $\operatorname{Str} M^{6}$ all vanishing.

Third, we emphasize that our definition of the string-theoretic supertraces in Eq. (四) is rooted in the actual string spectrum, and realizes the supertrace as an explicit sum over string states. As such it is completely general, and applies to large classes of tachyon-free four-dimensional string theories. By contrast, alternate supertrace calculations [7] consider only a particular family of non-SUSY string vacua which are continuously connected to a supersymmetric point, and define the supertraces through an expansion of $\Lambda_{\text {string }}$ with respect to the relevant SUSY-breaking parameter. Understanding the relation between these two approaches is an important issue.

Finally, we point out that an outstanding problem in string theory has been to understand the origins of misaligned SUSY as a symmetry, and to determine the kinds of dynamical SUSY-breaking scenarios which lead to such boson/fermion oscillations. Our results concerning the supertrace implications of misaligned SUSY will therefore be a useful tool in this quest.

\section{Acknowledgments}

One of us (KRD) thanks I. Antoniadis, C. Bachas, S. Chaudhuri, E. Kiritsis, C. Kounnas, D. Kutasov, C.S. Lam, J. Louis, and F. Zwirner for discussions, and the Technion ITP for hospitality during a visit when portions of this paper were written. RCM also thanks C. Burgess for discussions. This work was supported in 
part by DOE Grant No. DE-FG-0290ER40542, the US/Israel Bi-National Science Foundation, the Technion VPR Fund, NSERC (Canada), and FCAR (Québec).

\section{References}

[1] For a review, see: H.P. Nilles, Phys. Rep. 110 (1984) 1.

[2] D. Kutasov and N. Seiberg, Nucl. Phys. B358 (1991) 600.

[3] K.R. Dienes, hep-th/9402006, Nucl. Phys. B429 (1994) 533.

A non-technical introduction can also be found in:

K.R. Dienes, hep-th/9409114 (September 1994).

[4] G. Moore, Nucl. Phys. B293 (1987) 139;

K.R. Dienes, Phys. Rev. D42 (1990) 2004.

[5] K.R. Dienes, Phys. Rev. Lett. 65 (1990) 1979.

[6] K.R. Dienes and A.E. Faraggi (to appear).

[7] See, e.g., I. Antoniadis, C. Muñoz, and M. Quirós, Nucl. Phys. B397 (1993) 515 and references therein. 